九州大学学術情報リポジトリ

Kyushu University Institutional Repository

\title{
Confinement of charge carriers and molecular excitons within 5-nm-thick emitter layer in organic electroluminescent devices with a double heterostructure
}

\section{Adachi, Chihaya}

Department of Materials Science and Technology, Graduate School of Engineering Sciences, Kyushu University

\section{Tsutsui, Tetsuo}

Department of Materials Science and Technology, Graduate School of Engineering Sciences, Kyushu University

\section{Saito, Shogo}

Department of Materials Science and Technology, Graduate School of Engineering Sciences, Kyushu University

http://hdl. handle. net/2324/19441

出版情報: Applied Physics Letters. 57 (6), pp.531-533，1990-08-06. American Institute of Physics

バージョン :

権利関係: Copyright 1990 American Institute of Physics. This article may be downloaded for personal use only. Any other use requires prior permission of the author and the American Institute of Physics. 


\title{
Confinement of charge carriers and molecular excitons within 5-nm-thick emitter layer in organic electroluminescent devices with a double heterostructure
}

\author{
Chihaya Adachi, Tetsuo Tsutsui, and Shogo Saito \\ Department of Materials Science and Technology, Graduate School of Engineering Sciences, \\ Kyushu University, Kasuga-Shi. Fukuoka 816, Japan
}

(Received 29 January 1990; accepted for publication $30 \mathrm{May} 1990$ )

\begin{abstract}
Organic electroluminescent devices with a double-heterostructure indium-tin-oxide substrate/ hole transport layer/emitter layer/electron transport layer/MgAg have been fabricated by vacuum vapor deposition. The organic carrier transport and emitter layers were composed of amorphous films. In the doubleneterostructure devices, the luminance continued to lie in high level, even when the emitter thickness was $50 \AA$. The confnement of charge carriers and molecuiar excitons within a narrow emitter laye: was achieved.
\end{abstract}

Recent progress in high performance organic electroluminescent (EL) devices owes to the use of multilayer cell structures which are composed of an emitter layer (EML) and carrier transport layers. ${ }^{i-7}$ Tang and VanSlyke ${ }^{1}$ reported that the combination of the EML which possessed electron transporting tendency with a hole transport layer (HTL) was essential for high luminance and high stability of EL cells. "We proposed a three-layer cell structure in which an EML was sandwiched between hole and electron transport layers based on the idea that an electron transport layer (ETL) as well as HTL should play a major role in the increase of EL efficiency, when a variety of emitter materials was employed. Due to a lack of adequate ETL materials, however, our preliminary test indicated that the introduction of ETL did not contribute to the enhance. ment of $\mathrm{EL}$ efficiency at that time. ${ }^{4}$ In the wide search of ETL materials, we found an outstanding electron transport material, an oxadiazole derivative. Judging from its molecular structure, it was not assumed to be a perfect electron conductor, but it really worked as an excellent $\mathbb{E} T L$ in $E E$ devices. Thus using this material we, for the first time, succeeded in fabricating the EL device having hole conductor as an EML."

Now, we have both $\mathrm{HTL}$ and ETL materials and are ready to construct ideal three-layer structure cells. It is expected that the confinement of holes and electrons within a thin EML is attained in the three layer cell, which we call a double heterostructure (DH) hereafter.

In this letter, we report the fabrication of high performance DH cels and compare those cells with conventional two-layer (single heterostructure) cells. The effect of the thickness of the EML on EL efficiencies demonstrated the realization of confinement of holes and electrons within the very narrow EML. This also indicates the effective confinement of molecular excitons produced by the recombination of holes and electrons within the EML.

Two types of EL cell structures, single heterostructures (SHs) and DHs, were used in our experiment as shown in Fig. 1. The organic materials used in the experiment are also shown in the figure. We used an aromatic diamine (TAD) as the hole transport material and an $o x$ -

adiazole derivative (PBD) as the electron transport material. For emitter material, iriphenylamine derivative (NSD) was ised. In a previous paper, ${ }^{6}$ we showed that the SH device with NSD and PBD layers gave high EL eflciency in the case when the thickness of EML and ETL was fixed at $500 \AA$. In this study, the thickness of $\mathrm{HTL}$ and ETL was fixed at $500 \AA$, and the thickness of EML was varied from 500 to $50 \mathrm{~A}$.

Organic layers were deposited on a precleaned indiumtin-oxide (TTO) glass substrate and a cathode Mg $\mathrm{g}$ layer was deposited on ETL by codeposition in a vacuum of $10^{-7}$ Forr at room temperature. The thickness of the ITO layer was 1000 and sheet resistance was about $20 \Omega / 0$. The deposition rate for organic layers was about $2 \AA / s$. The thickness of organic layers was determined from frequency shifts on a quartz oscillating thickness monitor

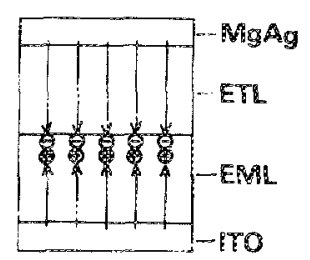

SH

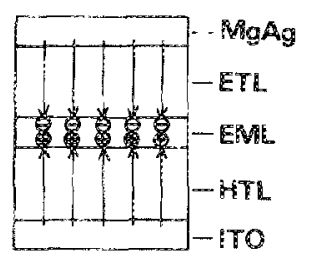

$\underline{\mathrm{DH}}$

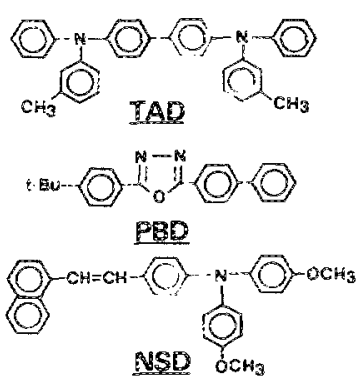

FiG. 1. Structures of the EL devices and the molecular structures of the materials used for the devices. 


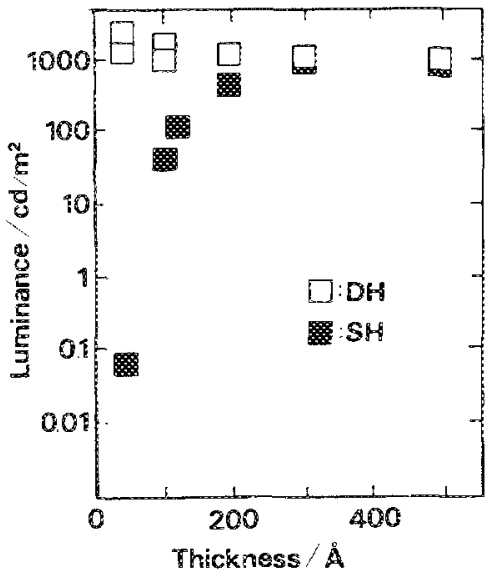

FIG. 2 Luminance at the current density of $100 \mathrm{~mA} /$ $\mathrm{cm}^{2}$ as a function of emitter thickness in $\mathrm{DH}$ and $\mathrm{SH}$ cells; (a) ITO/TAD/NSD/ PBD/MgAg, and (b) ITO/ NSD/PED/MgAg.

which was placed very close to an organic film on the substrate in the vacuem deposition chamber. The emitting area in EL cells was $0.2 \times 0.2 \mathrm{~cm}^{2}$. All of the deposited organic films were found to be amorphous based on the observation under a polarizing microscope.

Figure 2 shows the thickness dependence of luminance at a fixed current density of $100 \mathrm{~mA} / \mathrm{cm}^{2}$ for the $\mathrm{DH}$ and $S$ I cells. When the emitter thickness was $500 \AA$, the luminance of $1000 \mathrm{~cd} / \mathrm{m}^{2}$ was observed in both of the $\mathrm{SH}$ and $\mathrm{DH}$ cells. The $\mathrm{SH}$ cell structure gave a ingh $\mathrm{EL}$ effeiency, and there was no effect of the insertion of HTL (DH). However, when the thickness of the emitter layer was reduced to less than $300 \AA$, new feature appeared. The $\$$ cell showed a drastic decrease in luminance with the decrease of the emitter thickness. In contrast, constant high luminance was retained in the wide range of the emitter thicknesses in the $\mathrm{DH}$ cell. The Uuminance continued to lie in high level, even when the emitter thickness was reduced to $50 \mathrm{~A}$. The uniform emission indicates the uniformity of the ultrathin emitter layer.

Figure 3 shows the EL spectrum of the DH cell with $50 \AA$ emitter thickness at the current density of $10 \mathrm{~mA}$ $\mathrm{cm}^{2}$. Also, the photoluminescence (PL) spectra of NSD, FAD, and PBD layers are included. The EL spectrum corresponded exactly to the PL spectrum of the NSD firm. No emission from the TAD or the PBD layer was observed. This indicates that the site of carrier recombination located only within the EML. In other words, the confinement of charge carriers within the EML is attained; electrons injected from the $\mathrm{BDD}$ layer into the NSD layer are blocked at the TAD/NSD boundary, and holes injected from the TAD layer into the NSD layer are blocked at the NSD/BBD boundary.

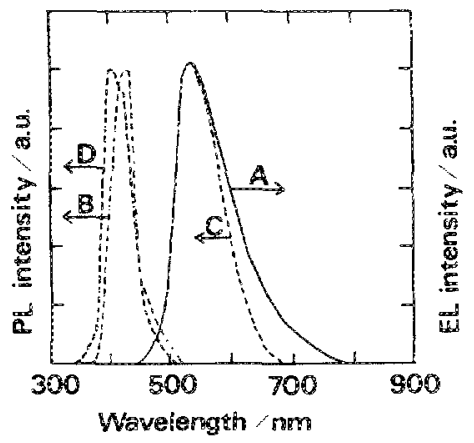

FIG. 3. EL spectrum of the $\mathrm{DH}$ cell and $\mathrm{PL}$ spectra of TAD, NSD, and PBD layers; (a) EL spectrum in a [TYO/ $\operatorname{TAD}(500 \AA) / \operatorname{NSD}(50 \AA)$ PBD $(500 \AA) / M g A g I$ cell, (b) $\mathrm{PL}$ specirum in a $\mathrm{EAD}$ film, (c) PL spectrum in a NSD film, and (d) PL spectrum in a PBD film.
The comparison of EL and PL spectra also gives the evidence of the confinement of molecular excitcns within the EML. The carrier transport materials, TAD and PBD, which possess emission peaks at around $400 \mathrm{~mm}$, are assumed to have exciton energies larger than that of the NSD layer. Thus, the excitons created within the EML have no ability to migrate into TAD or PBD layers. These considerations lead to the conclusion that the confinement of both charge carriers and molectar excitons within an EML was achieved even in the $50-\mathrm{A}$-thick EML.

Here, we would like also to mention another advantage of the use of the OH, that is, the insertion of ETL or HTL between EML and electrodes because it serves to prevent the quenching of excitons at electrode surfaces. ${ }^{8,9}$ According to our separate experiment, the $\mathrm{PL}$ intensity from the $\mathrm{EML}$ in a $\mathrm{ITO} / \mathrm{TAD}(200 \AA) / \mathrm{NSD}(25 \AA) / \mathrm{BDD}(200$ A)/MgAg cell was six times larger than that in an ITO/ $\mathrm{TAD}(200 \AA) / \mathrm{NSD}(25 \AA) / \mathrm{MgAg}$ cell. Evidently, the in serted PBD layer prevented the quenching of the molecu. lar excitors produced by photoexcitation. This result supports the assumption that the insertion of the carrier transport layers contribute to prevent the excitons from quenching at the electrode surfaces. This effect is particularly important, when EML thickness becomes narrower than the average migration length of excitons.

Based on the above considerations, we can point out two major reasons for the lower $\mathrm{EL}$ efficiency in the $\mathrm{SH}$ cells with EML at the thickness less than $300 \AA$. First, a large portion of electrons injected from the PBD layer pass through the EML without the encounter with holes. Second, a significant portion of the molecular excitons produced within EML reach the ITO electrode during a migration process and are quenched.

It should be stressed that the success in the construction of the DH with extremely thin EML stimulates our EL study towards exciting possibility in organic thin-film devices. The increase of the density of charge carriers and molecular excitons within extremely thin EML can be attained by means of the DH. Even the use of a single molecular layer for $\mathrm{EML}$ may be possible. The increase of excited states within a very narrow region opens the prospect for the attainment of population inversion of excited states.

The second prospect is concerned with the fabrication of molecular size devices. We deal with the EML thickness of about $50 \AA$, which corresponds to the stack of about ten emitter molecules. Therefore, our EL devices now work at the molecular size at least along the direction of applied field. We have obtained a way to observe electronic and optical phenomena on a few molecules sandwiched between fat and smooth organic carrier injection layers. This approach, we believe, gives one of the promising entrances towards the investigation of molecular size electronic devices.

We would like to acknowledge Mr. M. Hashimoto and Mr. M. Ohta in RICOH Co. Ltd. Japan for preparing the TAD and the NSD.

'C. W. Tang and S. A. VanSiyke, Appl. Mhys. Lett. 51, 913 (1987). 
${ }^{2}$ C. Adachi, S. Tokìto, T. Tsutsui, and S. Saito, Jpn. J. Appl. Phys. 27, L269 (1988).

${ }^{3}$ C. Adachi, S. Tokito, T. Tsutsui, and S. Saito, Jpn. J. Appl. Phys. 27, L713 (1988).

${ }^{4}$ C. Adachi, M. Morikawa, S. Tokito, T. Tsutsia, and S. Sairo, Proceedings of the 4ih International Workshop on Electroluminescence, October 11-14, 1988, Tottori, Japan, Springer Proceedings in Physics (Springer, Berlin, 1989), Vol. 38 , p. 358.
${ }^{5}$ C. W. Tang, S. A. VanSlyke, and C. H. Chen, J. Appl. Phys. 65, 3610 (1989).

${ }^{6} \mathrm{C}$. Adachi, T. Tsutsui, and \$. Saito, Appl. Phys. Lett. 55, 1489 (1989). ${ }^{7}$ C. Adachi, T. Tsutsui, and S. Saito, Appl. Phys. Lett. 5\$, 799 (1990).

${ }^{\mathrm{K}} \mathrm{K}$. C. Kao and W. HWang, Elecirical Transport in Solids (Pergamon, New York, 1981), p. 418.

${ }^{9} \mathrm{H}$. Kurcewski and H. Bässier, J. Lumines. 15, 26! (1977). 